\title{
A RANDOM GRAPH WITH A SUBCRITICAL NUMBER OF EDGES
}

\author{
B. PITTEL
}

\begin{abstract}
A random graph $G_{n}($ prob(edge) $=p)(p=c / n, 0<c<1)$ on $n$ labelled vertices is studied. There are obtained limiting distributions of the following characteristics: the lengths of the longest cycle and the longest path, the total size of unicyclic components, the number of cyclic vertices, the number of distinct component sizes, and the middle terms of the component-size order sequence. For instance, it is proved that, with probability approaching $(1-c)^{1 / 2} \exp \left(\sum_{j=1}^{l} c^{j} / 2 j\right)$ as $n \rightarrow \infty$, the random graph does not have a cycle of length $>l$. Another result is that, with probability approaching 1 , the size of the $\nu$ th largest component either equals an integer closest to $a \log \left(b n / \nu \log ^{5 / 2} n\right), a=a(c), b=b(c)$, or is one less than this integer, provided that $\nu \rightarrow \infty$ and $\nu=o\left(n / \log ^{5 / 2} n\right)$.
\end{abstract}

1. Introduction, results, notes. In the papers $[8,9]$ Erdős and Rényi began a systematic study of a random graph $G(n, M)$. In this model, the sample space is the set of all graphs on a vertex set $V=\{1, \ldots, n\}$ with exactly $M$ edges, and it is assumed that all such graphs are equally likely. There had been known another model of a random graph in which the number of edges of a sample graph is not fixed and a graph is assigned the probability

$$
p^{l} q^{\left(\begin{array}{c}
n \\
2
\end{array}\right)-l} \quad(q \geq 0, p \geq 0, p+q=1),
$$

if it has $l$ edges. Following the accepted notations, we denote this model $G(n, p)$. Thus, the random graph $G(n, p)$ has (does not have) an edge $(i, j), i, j \in V(i \neq j)$, with probability $p(q=1-p)$, and all $\left(\begin{array}{c}n \\ 2\end{array}\right)$ events "there is an edge $(i, j)$ " are mutually independent. It was indicated in [9], however, that the asymptotic behavior of these two models is essentially the same if $p\left(\begin{array}{c}n \\ 2\end{array}\right)=M$, which means simply that the average number of edges in $G(n, p)$ is precisely $M$. Of course, it should be required, in addition, that $p$ depends on $n$ in such a way that, with probability approaching 1 as $n \rightarrow \infty$-in short, almost surely (a.s.) - the random number of edges in $G(n, p)$ is relatively close to its expected value. In many instances, this heuristic principle can be rigorously justified and used to get statements about $G(n, M)$ as direct corollaries of the results for $G(n, p)$, Bollobás [6], Pittel [14]. For example, it was proven in [14] that, for every set $A$ of graphs with $M$ edges,

$$
P(G(n, M) \in A)=o(1) \quad \text { if } \quad P(G(n, p) \in A)=o\left[(q M)^{-1 / 2}\right],
$$

$\left(p\left(\begin{array}{l}n \\ 2\end{array}\right)=M\right)$ and $q M \rightarrow \infty$.

In [9], a particular attention was paid to a case when $M=a n$, where $a$ is a fixed constant. It was discovered that the asymptotic structure of the graph $G(n, M)$

Received by the editors July 24, 1986.

1980 Mathematics Subject Classification (1985 Revision). Primary 05C80, 05C30, 60C05, 69F99. 
strongly depends on the value of $a$. Namely, for $a<1 / 2$, a.s. all the vertices belong to components which are trees, except a bounded, in probability, number of vertices which belong to unicyclic components; furthermore the size of the largest component is relatively close to $A \log n, A=A(a)$. If $a>1 / 2$, then a.s. $G(n, M)$ has a unique giant component whose size depends linearly on $n$. As for the rest of the graph, it still consists of many tree-components with the largest one having size $\approx A \log n$, and it may also have a bounded, in probability, number of vertices which comprise unicyclic components, Bollobás [6]. Furthermore, Erdős and Rényi proved in [9] that, at a critical point $a=1 / 2$, the largest tree-component of $G(n, M)$ has a.s. a size of order $n^{2 / 3}$, which is sandwiched between $\log n$ and $n$, the orders of sizes of the largest components resp. for $a<1 / 2$ and $a>1 / 2$. Also, according to Bollobás [6], the largest component is a.s. at most of order $(\log n)^{1 / 2} n^{2 / 3}$, thus not much larger than the largest tree-component.

Now, the average number of edges in the random graph $G(n, p)$ grows as an if $p \sim c / n, c=2 a$. So, it should not come as a surprise that the above cited statements have their analogues for the graph $G(n, p), p=c / n$, with the critical value $c=1$.

Our goal in this paper is to obtain the limiting distributions of several characteristics of the graph $G(n, p), p=c / n$, in a subcritical range, that is for $c<1$. Some of our statements cover the supercritical range $c>1$ as well (Lemmas 2, 5, (2.26), (2.27)).

Specifically, we study (1) $\mathscr{C}_{n}$, the length of the longest cycle, (by convention, $\mathscr{C}_{n}=0$ if there are no cycles); (2) $\mathscr{P}_{n}$, the length of the longest path; (3) $\mathscr{U}_{n}$ and $\mathscr{V}_{n}$, respectively, the total number of vertices in the unicyclic components and the total number of vertices forming the cycles in these components; (4) $\mathscr{D}_{n}$, the number of all distinct component sizes; and (5) $\mathscr{S}_{n \nu}$, the size of the $\nu$ th largest component $(\nu \rightarrow \infty)$.

Note. Since $c<1$, a.s. every component of $G(n, p)$ is either a tree or unicyclic, so that a.s. $\mathscr{U}_{n}$ is the total number of vertices which belong to the nontree components, and $\mathscr{V}_{n}$ is the total length of all the cycles.

To formulate our results, we need a function $\tau(x)$ which is the exponential generating function of a sequence $\{t(\kappa): \kappa \geq 1\}$, where $t(\kappa)$ is the total number of rooted trees on $\kappa$ labelled vertices, that is $\tau(x)=\sum_{\kappa \geq 1} x^{\kappa} t(\kappa) / \kappa$ !. It is well known that $t(\kappa)=\kappa^{\kappa-1}$ (Cayley), that the series converges for $|x| \leq e^{-1}$ and, for those $x$ 's, $\tau(x)$ is a solution of a transcendental equation

$$
\tau=x e^{\tau}
$$

(see Moon [12], who attributes this result to Dziobek (1917), Polya (1937) and Bol (1938)). Another related function used below is $\tau_{h}(x)=\sum_{\kappa \geq 1} x^{\kappa} t(\kappa, h) / \kappa$ !, where $t(\kappa, h)$ is the total number of rooted trees with height $\leq h$. (The height of a rooted tree is defined as the length of the longest path in the tree which begins at the root.) Using a recurrence relation

$$
\tau_{h+1}(x)=x \exp \left[\tau_{h}(x)\right], \quad \tau_{0}(x)=x, \quad h \geq 0,
$$

due to Riordan [18], we will prove that for every $x \in\left(0, e^{-1}\right)$, there exists $\gamma=\gamma(x)$ such that

$$
\tau_{h}(x)=\tau(x)-\gamma \tau^{h}(x)-\left[\gamma^{2} \tau^{2 h}(x) / 2(1-\tau(x))\right](1+o(1)), \quad h \rightarrow \infty .
$$


So, let $c<1$. We begin with

THEOREM 1. For every fixed integer $l \geq 2$,

(a)

$$
P\left(\mathscr{C}_{n} \leq l\right) \rightarrow(1-c)^{1 / 2} \exp \left(\sum_{j=1}^{l} \frac{c^{j}}{2 j}\right), \quad n \rightarrow \infty,
$$

whence, putting $l=2$,

(b)

$$
P(G(n, p) \text { does not have a cycle }) \rightarrow(1-c)^{1 / 2} \exp \left(c / 2+c^{2} / 4\right) .
$$

Note. The relation (1.4) was stated in [9] for the random graph $G(n, M)$; however, a brief argument intended to prove it contained a gap which is very difficult, in our opinion, to close. Our proof uses a different approach.

Next

THEOREM 2. Given an integer d, define $y$ by

$$
d=(\log n+y) / \log (1 / c) \text {. }
$$

If $d \rightarrow \infty$, as $n \rightarrow \infty$, in such a way that $y$ is bounded, then

$$
P\left(\mathscr{P}_{n}<d\right)-\exp \left(-\beta e^{-y}\right) \rightarrow 0
$$

here

$$
\beta=\bar{\gamma}^{2}(1-c) / 2 c^{4}, \quad \bar{\gamma}=\gamma\left(c e^{-c}\right),
$$

see (1.2).

Note. Thus, somewhat imprecisely,

$$
\mathscr{P}_{n}=\left(\log n+\mathscr{Q}_{n}\right) / \log (1 / c),
$$

where $\mathscr{Q}_{n}$ is double exponentially distributed in the limit.

We should also notice that, for $c>1$, the behavior of $\mathscr{C}_{n}$ and $\mathscr{P}_{n}$ is strikingly different: a.s. both of them are of magnitude $n$ (Ajtai, Komlós, Szemerédi [1] , de la Vega [21], Bollobás [4], Bollobás, Fenner, Frieze [5]). On the other hand, according to Bollobás [6, Chapter $\mathrm{X}$ ], the diameter of the giant component of $G(n, p), p=c / n, c>1$, is a.s. relatively close to $\log _{c} n$ provided that $c$ is large enough.

Turn now to the terms $\mathscr{S}_{n \nu}, \nu \geq 1$, of the component-size order sequence. By a theorem of Erdős and Rényi [9] (see also Bollobás [6, Chapter 6]), for every fixed $\nu \geq 1$,

$$
\mathscr{S}_{n \nu}=\alpha^{-1}\left[\log n-\frac{5}{2} \log \log n\right]+O_{p}(1),
$$

where $\alpha=c-1-\log c$, and $O_{p}(1)$ stands for a random variable bounded in probability as $n \rightarrow \infty$.

Our next theorem characterizes the asymptotic behavior of the "middle" terms $\mathscr{S}_{n \nu}$, i.e., of those with $\nu \rightarrow \infty$. 
THEOREM 3. Introduce a sequence

$$
s_{n \nu}=\alpha^{-1} \log \left(n \delta / \nu \log ^{5 / 2} n\right), \quad \nu \geq 1,
$$

so that

$$
s_{n \nu}=s_{n 1}-\alpha^{-1} \log \nu, \quad \nu \geq 1
$$

where

$$
\delta=(2 \pi)^{-1 / 2} c^{-1}\left(1-e^{-\alpha}\right)^{-1} \alpha^{5 / 2} .
$$

If $\nu \rightarrow \infty, \nu=o\left(n / \log ^{5 / 2} n\right)(n \rightarrow \infty)$, then a.s. $\mathscr{S}_{n \nu}$ equals either $\left\langle s_{n \nu}\right\rangle$ or $\left\langle s_{n \nu}\right\rangle-1(\langle x\rangle$ denotes an integer closest to $x)$.

Note. Comparing (1.5) and the last theorem, we can see that in both cases $\Delta \mathscr{S}_{n \nu}=\mathscr{S}_{n \nu}-s_{n \nu}$ is bounded in probability. A difference is that, for $\nu$ fixed, $\Delta \mathscr{S}_{n \nu}$ has a double exponential distribution in the limit [9], while for $\nu \rightarrow \infty$ the probable range of $\Delta \mathscr{S}_{n \nu}$ consists of only two numbers. In fact, the argument we use to prove this result yields also if, in addition, the fractional part of $s_{n \nu}$ is bounded away from both 0 and 1 then a.s. $\mathscr{S}_{n \nu}$ equals $\left[s_{n \nu}\right]$, the integer part of $s_{n \nu} \cdot\left(\left\langle s_{n \nu}\right\rangle-1 \leq\left[s_{n \nu}\right] \leq\left\langle s_{n \nu}\right\rangle \cdot\right)$

Besides the individual terms of the sequence $\left\{\mathscr{S}_{n \nu}, \nu \geq 1\right\}$, one may also be interested in its properties as a whole. One such a characteristic is $\mathscr{D}_{n}$, the number of all distinct component sizes. The problem of determining the asymptotic behavior of $\mathscr{D}_{n}$ is a particular instance of a more general problem for partition-type combinatorial schemes posed by Wilf [22]. (His paper contains solutions of the corresponding problems for cycles of a random permutation, and summands of a random partition of a large integer.)

To formulate our result, it is necessary to introduce $\mathscr{D}_{n}^{\prime}$ and $\mathscr{D}_{n}^{\prime \prime}$, which are respectively the number of all distinct sizes of tree-components and unicyclic components. (Since $c<1, \mathscr{D}_{n}=\mathscr{D}_{n}^{\prime}+\mathscr{D}_{n}^{\prime \prime}$ a.s. when $n \rightarrow \infty$.) Let us also agree to write $X_{n} \approx Y_{n}\left(X_{n} \Rightarrow Y\right), n \rightarrow \infty$, if $X_{n}, Y_{n} \in R^{\kappa}\left(Y \in R^{\kappa}\right)$ are the random vectors such that

$$
\begin{gathered}
E\left[\exp \left(i v^{T} X_{n}\right)\right]-E\left[\exp \left(i v^{T} Y_{n}\right)\right] \rightarrow 0, \quad \forall v \in R^{\kappa}, \\
\left(E\left[\exp \left(i v^{T} X_{n}\right)\right] \rightarrow E\left[\exp \left(i v^{T} Y\right)\right]\right),
\end{gathered}
$$

when $n \rightarrow \infty$. (The second relation holds, of course, iff $X_{n}$ converges to $Y$ in distribution.)

THEOREM 4. Introduce $D_{n}$ and $d_{n}$, respectively the integer part and the fractional part of $\alpha^{-1} \log \left[\alpha^{5 / 2} n / c(2 \pi)^{1 / 2} \log ^{5 / 2} n\right]$. When $n \rightarrow \infty$,

$$
\left(\mathscr{D}_{n}^{\prime}-\mathscr{D}_{n}, \mathscr{D}_{n}^{\prime \prime}\right) \approx\left(\mathscr{E}_{n}^{\prime}, \mathscr{E}_{n}^{\prime \prime}\right)
$$

here

$$
\mathscr{E}_{n}^{\prime}=\sum_{j=-\infty}^{\infty} \varepsilon_{n j}^{\prime}, \quad \mathscr{E}_{n}^{\prime \prime}=\sum_{j=3}^{\infty} \varepsilon_{n j}^{\prime \prime},
$$

$\varepsilon_{n j}^{\prime} \in\{0,1\}$ for $j \leq-1, \varepsilon_{n j}^{\prime} \in\{-1,0\}$ for $j \geq 0, \varepsilon_{n j}^{\prime \prime} \in\{0,1\}, j \geq 3$, and all the $\varepsilon$ 's are independent. More precisely,

$$
\begin{aligned}
& P\left(\varepsilon_{n j}^{\prime}=0\right)=\exp \left[-e^{\alpha\left(d_{n}+j\right)}\right], \quad j \leq-1, \\
& P\left(\varepsilon_{n j}^{\prime}=-1\right)=\exp \left[-e^{\alpha\left(d_{n}+j\right)}\right], \quad j \geq 0, \\
& P\left(\varepsilon_{n j}^{\prime \prime}=0\right)=\exp \left[-u(j)\left(c e^{-c}\right)^{j} / j !\right], \quad j \geq 3,
\end{aligned}
$$


where $u(j)$ is the total number of connected unicyclic graphs on $j$ labelled vertices. (According to Katz [11] and Rényi [16],

$$
\left.u(j)=\frac{1}{2} \sum_{\kappa=3}^{j}(j)_{\kappa} j^{j-\kappa-1}, \quad u(j) \sim(\pi / 8)^{1 / 2} j^{j-1 / 2}, \quad(j \rightarrow \infty) .\right)
$$

Note. Since both $\mathscr{E}_{n}^{\prime}$ and $\mathscr{E}_{n}^{\prime \prime}$ are bounded in probability, it follows from this theorem that

$$
\begin{aligned}
& \mathscr{D}_{n}^{\prime}=D_{n}+O_{p}(1), \quad n \rightarrow \infty, \\
& \mathscr{D}_{n}^{\prime \prime}=O_{p}(1), \quad n \rightarrow \infty .
\end{aligned}
$$

Clearly, the second relation should be expected since even the total number of all unicyclic components is $O_{p}(1)$. Far more interesting is the first relation; indeed, in view of (1.5), it means that the total number of integers from 1 to $\mathscr{S}_{n 1}$ (the size of the largest component), which are not component sizes, is bounded in probability.

(Another instance of a partition-type scheme with this property is the random partition of an $n$-element set. Sachkov [19] proved that the size of the largest block grows, in probability, as $e \log n$ when $n \rightarrow \infty$. Quite recently, answering a question posed by Wilf [22], Odlyzko and Richmond [13] proved that in probability, and on average, the total number of distinct block sizes in the random partition is asymptotic to $e \log n$, too.)

It remains to consider $\mathscr{U}_{n}$ and $\mathscr{V}_{n}$ which are, as we remember, respectively the total size of all unicyclic components and the total length of cycles in them.

THEOREM $5 . \mathscr{U}_{n} \Rightarrow \mathscr{U}, \mathscr{V}_{n} \Rightarrow \mathscr{V}$, where

$$
\begin{aligned}
& E\left(z^{\mathscr{U}}\right)=f(c) / f\left[\tau\left(z c e^{-c}\right)\right], \quad|z|<e^{\alpha}, \\
& E\left(z^{\mathscr{V}}\right)=f(c) / f(z c), \quad|z|<c^{-1},
\end{aligned}
$$

and

$$
f(x) \underset{\text { def }}{=}(1-x)^{1 / 2} \exp \left(x / 2+x^{2} / 4\right), \quad|x|<1
$$

Moreover,

$$
E\left(\mathscr{U}_{n}^{\kappa}\right) \rightarrow E\left(\mathscr{U}^{\kappa}\right), \quad E\left(\mathscr{V}_{n}^{\kappa}\right) \rightarrow E\left(\mathscr{V}^{\kappa}\right), \quad \kappa \geq 1(n \rightarrow \infty)
$$

so, in particular,

$$
\begin{aligned}
& E\left(\mathscr{U}_{n}\right) \rightarrow c^{2} / 2(1-c)^{2}, \quad \operatorname{var}\left(\mathscr{U}_{n}\right) \rightarrow c^{3}(3-c) / 2(1-c)^{4} \quad(n \rightarrow \infty), \\
& E\left(\mathscr{V}_{n}\right) \rightarrow c^{3} / 2(1-c), \quad \operatorname{var}\left(\mathscr{V}_{n}\right) \rightarrow c^{3}\left(3-c-c^{3}\right) / 2(1-c)^{2} \quad(n \rightarrow \infty) .
\end{aligned}
$$

The formula for $\lim _{n \rightarrow \infty} E\left(\mathscr{V}_{n}\right)$ was obtained in [9]; one can also find in [9 and 6] the infinite series-type formulae for $\lim E\left(\mathscr{U}_{n}\right)$ and $\lim \operatorname{var}\left(\mathscr{U}_{n}\right)$.

$A$ final note. Presently, we are studying the limiting distributions for the critical case when $c=1$, or $c$ approaches 1 as $n \rightarrow \infty$. Among the results is the following:

$P(G(n, 1 / n)$ has a component which is neither a tree nor unicyclic)

$\rightarrow 1-(2 / 3)^{1 / 2}=0.183 \ldots$

as $n \rightarrow \infty$. (It is worth noticing that the limiting values of the corresponding probability for $c<1$ and $c>1$ are 0 and 1 respectively.) It shows that $G(n, 1 / n)$ is planar with probability bounded away from 0 as $n \rightarrow \infty$, which was conjectured by Erdős and Rényi in [9] in an equivalent case of the graph $G(n, M), M \sim n / 2$. 


\section{Proofs.}

PROOF OF THEOREM 1. Denote the number of all unicyclic connected graphs on $\kappa$ (labelled) vertices, whose cycle has length more than $l$, by $u(\kappa, l)(\kappa>l \geq 2)$. Clearly, $u(\kappa, 2)=u(\kappa)$ which is the number of all unicyclic connected graphs on $\kappa$ vertices. It is known (Wright [23]) that, for $|x|<e^{-1}$,

$$
u(x) \underset{\operatorname{def}}{=} \sum_{\kappa \geq 3} \frac{x^{\kappa} u(\kappa)}{\kappa !}=\log [1-\tau(x)]^{-1 / 2}-\frac{\tau(x)}{2}-\frac{\tau^{2}(x)}{2} ;
$$

here $\tau(x)$ is the exponential generating function of the sequence $\{t(\kappa): \kappa \geq 1\}$, $t(\kappa)=\kappa^{\kappa-1}$ being the number of all rooted trees on $\kappa$ vertices. Extending Wright's argument, we prove

LEMMA 1. For $|x|<e^{-1}$,

$$
\begin{aligned}
u_{l}(x) & \underset{\operatorname{def}}{ } \sum_{\kappa>l} \frac{x^{\kappa} u(\kappa, l)}{\kappa !} \\
& =\log [1-\tau(x)]^{-1 / 2}-\sum_{j=1}^{l} \frac{\tau^{j}(x)}{2 j} .
\end{aligned}
$$

PROOF. First of all,

$$
u(\kappa, l)=\sum_{j=l+1}^{\kappa} 2^{-1}(j-1) ! F(\kappa, j),
$$

where $F(\kappa, j)$ is the total number of forests of $j$ rooted trees on $\kappa$ vertices, because $2^{-1}(j-1)$ ! is the number of ways for the roots of $j$ trees to form an unoriented cycle. Second,

$$
F(\kappa, j)=(j !)^{-1} \sum \kappa ! \prod_{s=1}^{j} \frac{t\left(\kappa_{s}\right)}{\kappa_{s} !},
$$

where the sum is taken over $j$-tuples $\left(\kappa_{1}, \ldots, \kappa_{j}\right)$ such that $\kappa_{1} \geq 1, \ldots, \kappa_{j} \geq 1$ and $\kappa_{1}+\cdots+\kappa_{j}=\kappa$. Subsequently,

$$
F(\kappa, j)=(\kappa ! / j !) \operatorname{coeff}_{x^{\kappa}}\left[\tau^{j}(x)\right],
$$

or

$$
\begin{aligned}
\frac{u(\kappa, l)}{\kappa !} & =\sum_{j=l+1}^{\kappa} \operatorname{coeff}_{x^{\kappa}} \frac{\left[\tau^{j}(x)\right]}{2 j} \\
& =\operatorname{coeff}_{x^{\kappa}} \sum_{j \geq l} \frac{\tau^{j}(x)}{2 j},
\end{aligned}
$$

and

$$
\begin{aligned}
u_{l}(x) & =\sum_{j>l} \frac{\tau^{j}(x)}{2 j}=\sum_{j \geq 1} \frac{\tau^{j}(x)}{2 j}-\sum_{j=1}^{l} \frac{\tau^{j}(x)}{2 j} \\
& =\log [1-\tau(x)]^{-1 / 2}-\sum_{j=1}^{l} \frac{\tau^{j}(x)}{2 j} .
\end{aligned}
$$


Next

LEMMA 2. Let $c \neq 1$; introduce the random variable $X_{n l}$ which is the total number of unicyclic components of the graph $G(n, p), p=c / n$, whose cycle contains more than $l$ vertices. $X_{n l}$ converges in distribution, and in terms of all moments, to a Poisson distributed random variable with parameter $\lambda$ (in short, $X_{n l} \stackrel{m}{\Rightarrow} P(\lambda)$ ), where

$$
\lambda=\log (1-\bar{\tau})^{-1 / 2}-\sum_{j=1}^{l} \frac{\bar{\tau}^{j}}{2 j},
$$

and $\bar{\tau}$ is the only root of the equation $\tau e^{-\tau}=c e^{-c}$ subject to restriction $\tau<1$. In particular,

$$
P\left(\mathscr{C}_{n} \leq m\right)=P\left(X_{n}=0\right) \rightarrow e^{-\lambda}=(1-\bar{\tau})^{-1 / 2} \exp \left(\sum_{j=1}^{l} \frac{\bar{\tau}^{j}}{2 j}\right) .
$$

Theorem 1 will follow at once since $\bar{\tau}=c$ for $c<1$.

PROOF OF LEMMA 2. Since $\left(X_{n l}\right)_{r}=X_{n l}\left(X_{n l}-1\right) \cdots\left(X_{n l}-r+1\right)$ is the total number of ways to select $r$ components in question with order and without replacement, we have

$$
\begin{aligned}
E\left[\left(X_{n l}\right)_{r}\right]= & \sum\left(\begin{array}{c}
n \\
i_{1} \cdots i_{r}
\end{array}\right) \prod_{s=1}^{r} u\left(i_{s}, l\right) p^{i_{s}} q^{\left(i_{2}\right)-i_{s}+\left(n-i_{s}\right) i_{s}} \\
& \cdot \prod_{1 \leq s \neq s^{\prime} \leq r} q^{-i_{s} i_{s^{\prime}} / 2} \quad(q=1-p) .
\end{aligned}
$$

Here

$$
\left(\begin{array}{c}
n \\
i_{1} \cdots i_{r}
\end{array}\right)=n ! / \prod_{s=1}^{r+1} i_{s} !, \quad i_{r+1}=n-i, i=i_{1}+\cdots+i_{r}
$$

and the sum is taken over all tuples $\left(i_{1}, \ldots, i_{r}\right)$ such that $i_{s}>l(1 \leq s \leq r)$. Since similar relations will appear again later, let us explain (2.2) in detail. First,

$$
\left(\begin{array}{c}
n \\
i_{1} \cdots i_{r}
\end{array}\right)
$$

is the number of ways to select (with order) $r$ disjoint sets of vertices having cardinalities $i_{1}, \ldots, i_{r}$, and $i_{r+1}$ is the number of the remaining vertices. Second,

$$
\prod_{s=1}^{r} u\left(i_{s}, l\right) p^{i_{s}} q^{\left(i_{s}\right)-i_{s}}
$$

is the probability that each of the first $r$ subsets of vertices induces a unicyclic connected subgraph of $G(n, p)$ whose cycle is of length $>l$. Third,

$$
\prod_{s=1}^{r} q^{\left(n-i_{s}\right) i_{s}} \cdot \prod_{1 \leq s \neq s^{\prime} \leq r} q^{-i_{s} i_{s^{\prime}} / 2}=\prod_{1 \leq s \neq s^{\prime} \leq r+1} q^{i_{s} i_{s^{\prime}} / 2}
$$

is the probability that all $(r+1)$ induced subgraphs are isolated.

If we show that $E\left[\left(X_{n l}\right)_{r}\right] \rightarrow \lambda^{r}, r \geq 1$, it will follow that $X_{n l} \stackrel{m}{\Rightarrow} P(\lambda)$, since $\lambda^{r}$ is the $r$ th factorial moment of $P(\lambda)$. The argument we suggest uses some ideas of 
the proof of a theorem in [6, Chapter V, §2]. Break up the sum in (2.2) into the sums $\Sigma^{\prime}$ and $\Sigma^{\prime \prime}$ taken over $r$-tuples $\left(i_{1}, \ldots, i_{r}\right)$ such that $\max _{1 \leq s \leq r} i_{s} \leq n_{1}$ and $\max _{1 \leq s \leq r} i_{s}>n_{1}$ respectively; here $n_{1}=\left[n^{1 / 3}\right]$. Consider first $\Sigma^{\prime}$. Denoting its common term by $C\left(i_{1}, \ldots, i_{r}\right)$, we have

$$
C\left(i_{1}, \ldots, i_{r}\right)=\left[\prod_{s=1}^{r}\left(c e^{-c}\right)^{i_{s}} \frac{u\left(i_{s}, l\right)}{i_{s} !}\right]\left(1+O\left(n^{-1 / 3}\right)\right),
$$

because

$$
\begin{aligned}
& \left(\begin{array}{c}
n \\
i_{1} \cdots i_{r}
\end{array}\right)=(n)_{i} / \prod_{s=1}^{r} i_{s} !=\left(n^{i} / \prod_{s=1}^{r} i_{s} !\right)\left(1+O\left(i^{2} / n\right)\right), \\
& q^{\left(\begin{array}{c}
i_{s} \\
2
\end{array}\right)-i_{s}+\left(n-i_{s}\right) i_{s}}=(1-c / n)^{n i_{s}}\left(1+O\left(i_{s}^{2} / n\right)\right)=e^{-c}\left(1+O\left(i_{s}^{2} / n\right)\right),
\end{aligned}
$$

and

$$
\prod_{1 \leq s \neq s^{\prime} \leq r} q^{-i_{s} i_{s^{\prime}} / 2}=1+O\left(i^{2} / n\right) .
$$

Hence, setting $x=c e^{-c}$,

$$
\begin{aligned}
\Sigma^{\prime} & =\left[\sum_{i=1}^{n_{1}} \frac{x^{i} u(i, l)}{i !}\right]^{r}\left(1+O\left(n^{-1 / 3}\right)\right) \\
& \rightarrow\left[u_{l}(x)\right]^{r}=\lambda^{r} \quad(n \rightarrow \infty) .
\end{aligned}
$$

It remains to prove that $\Sigma^{\prime \prime} \rightarrow 0$ as $n \rightarrow \infty$. According to (1.8),

$$
u(j, l) \leq u(j)=O\left(j^{j}\right) \quad(j \rightarrow \infty) .
$$

Introduce

$$
\tilde{\Sigma}^{\prime \prime}=\sum_{\max i_{s}>n_{1}} \tilde{C}\left(i_{1}, \ldots, i_{r}\right),
$$

where $\widetilde{C}(\cdot)$ is defined like $C(\cdot)$ except that each factor $u\left(i_{s}, l\right)$ is replaced by $i_{s}^{i_{s}}$. By (2.3), it suffices to prove that $\widetilde{\Sigma}^{\prime \prime} \rightarrow 0$ when $n \rightarrow \infty$.

To this end, notice that if $\max i_{s}=n_{1}$ then (cf. (2.3))

$$
\begin{aligned}
\widetilde{C}\left(i_{1}, \ldots, i_{r}\right) & =\left(\prod_{s=1}^{r} \frac{x^{i_{s}} i_{s}^{i_{s}}}{i_{s} !}\right)\left(1+O\left(n^{-1 / 3}\right)\right) \\
& =O\left[\left(x^{n_{1}} n_{1}^{n_{1}} / n_{1} !\right)^{\left|\left\{s: i_{s}=n_{1}\right\}\right|}\right] \\
& \leq b \rho^{n_{1}}, \quad \rho=e c e^{-c}, b>0 .
\end{aligned}
$$

Here $\rho<1$ since $c \neq 1$. Furthermore, for a fixed $\nu \in(1, \ldots, r)$,

$$
\begin{aligned}
\widetilde{C} & \left(i_{1}, \ldots, i_{\nu}+1, \ldots, i_{r}\right) / \widetilde{C}\left(i_{1}, \ldots, i_{\nu}, \ldots, i_{r}\right) \\
& =\left[(n-i) /\left(i_{\nu}+1\right)\right]\left[\left(i_{\nu}+1\right)^{i_{\nu}+1} / i_{\nu}^{i_{\nu}}\right]\left[p q^{n-i_{\nu}-2}\right]\left[\prod_{s \neq \nu} q^{-i_{s}}\right] \\
& =\left(1+1 / i_{\nu}\right)^{i_{\nu}} p(n-i) q^{n-i-2} \\
& \leq\{e p(n-i) \exp [-p(n-i)]\}(1-c / n)^{-2} \leq(1-c / n)^{-2} \leq(1-2 c / n)^{-1} .
\end{aligned}
$$


Combining (2.7) and (2.8) we get: if $\max _{1 \leq s \leq r} i_{s}>n_{1}$ then

$$
\begin{aligned}
\widetilde{C}\left(i_{1}, \ldots, i_{r}\right) & \leq b \rho^{n_{1}} \prod_{s: i_{s}>n_{1}}\left(1-\frac{2 c}{n}\right)^{-\left(i_{s}-n_{1}\right)} \\
& \leq b \rho^{n_{1}} \prod_{s=1}^{r}\left(1-\frac{2 c}{n}\right)^{-\left(i_{s}-n_{1}\right)} \\
& \leq b \rho_{1}^{n_{1}} \prod_{s=1}^{r}\left(1-\frac{2 c}{n}\right)^{-i_{s}}, \quad \rho_{1} \in(\rho, 1) .
\end{aligned}
$$

Hence,

$$
\tilde{\Sigma}^{\prime \prime} \leq b \rho_{1}^{n_{1}} \sum_{i_{1}, \ldots, i_{r} \geq 0} \prod_{s=1}^{r}\left(1-\frac{2 c}{n}\right)^{-i_{s}}=b \rho_{1}^{n_{1}}\left(\frac{n}{2 c}\right)^{r} \rightarrow 0
$$

$(n \rightarrow \infty)$.

Theorem 1 is proved.

Note. While proving Lemma 2, we showed, in particular, that for $c<1$

$$
E\left(X_{n 2}\right) \rightarrow \log (1-c)^{-1 / 2}-\left(c / 2+c^{2} / 4\right)
$$

where $X_{n 2}$ is the total number of all unicyclic components of $G(n, p)$. On the other hand, the expected number of all cycles in $G(n, p)$ is clearly (cf. [9])

$$
\begin{aligned}
\sum_{\kappa=3}^{n}\left(\begin{array}{l}
n \\
\kappa
\end{array}\right)\left[\frac{(\kappa-1) !}{2}\right] p^{\kappa} & \rightarrow \sum_{\kappa \geq 3} \frac{c^{\kappa}}{2 \kappa} \\
& =\log (1-c)^{-1 / 2}-\left(c / 2+c^{2} / 4\right) \quad(n \rightarrow \infty) .
\end{aligned}
$$

Combination of (2.10) and (2.11) provides an alternative proof of a known fact (see Introduction) that, for $c<1$, a.s. every component of $G(n, p)$ is either a tree or unicyclic. In fact, it shows also that the probability of this event is at least $1-$ const $n^{-w}, \forall w<1$.

Next,

PROOF OF THEOREM 2. First (Lemmas 3, 4), we obtain some results regarding asymptotic enumeration of trees with given height and diameter. Second (Lemma 5 ), we prove that the number of the tree-components of the graph $G(n, p), c \neq 1$, whose diameter exceeds a certain value, is asymptotically Poisson distributed. In case $c<1$, it implies Theorem 2 .

Let $t^{0}(\kappa, h)(t(\kappa, h))$ denote the total number of rooted trees on $\kappa$ vertices of height $=h(\leq h)$. Let $T^{0}(\kappa, d)(T(\kappa, d))$ denote the total number of unrooted trees on $\kappa$ vertices of diameter $=d(\geq d)$. Introduce the exponential generating functions $\tau_{h}^{0}(x), \tau_{h}(x), T_{d}^{0}(x)$ and $T_{d}(x)$ for these four sequences.

According to Riordan [18] (see also Rényi and Szekeres [17], Moon [12]),

$$
\tau_{h+1}(x)=x \exp \left[\tau_{h}(x)\right], \quad \tau_{0}(x)=x .
$$

Riordan also proved in [18] that

$$
\begin{aligned}
T_{d}^{0}(x) & =\left[\tau_{h}^{0}(x)\right]^{2} / 2, \quad \text { if } d=2 h+1, \\
& =\tau_{h}^{0}(x)-\tau_{h-1}(x) \tau_{h-1}^{0}(x), \quad \text { if } d=2 h .
\end{aligned}
$$


The strikingly beautiful relation (2.12) follows from an observation that every rooted tree of height $\leq h+1$ is obtained by taking several rooted trees of height $\leq h$ and joining their roots to a new vertex. As for (2.13), its derivation is based on the following. For $d=2 h+1$, there exists an obvious one-to-one correspondence between $\mathscr{T}_{d}^{0}$, the set of all trees of diameter $=d$, and the set of all unordered pairs of rooted trees each of height $=h$. For $d=2 h$, there is a one-to-one correspondence between $\mathscr{T}_{d}^{0}$ and the set of all rooted trees of height $=h$ such that there are at least two edges adjacent to the root which lead to vertices located at distance $h$ from the root.

There does not seem to exist a reasonably explicit formula for $\tau_{h}(x)$. However, in case $x \in\left(0, e^{-1}\right)$, it is possible to derive a sharp asymptotic formula for $\tau_{h}(x)$ $(h \rightarrow \infty)$, expressed-in the main-through powers of $\tau(x)$, the exponential generating function of all rooted trees. (In [17], a much more delicate analysis is done in case $x \rightarrow e^{-1}$ as $h \rightarrow \infty$, in order to obtain the limiting distribution of the height of the random tree.)

LEMMA 3. For every $x \in\left(0, e^{-1}\right)$, there exists $\gamma=\gamma(x)>0$ such that

$$
\tau_{h}(x)=\tau(x)-\gamma \tau^{h}(x)-\left[\gamma^{2} \tau^{2 h}(x) / 2(1-\tau(x))\right][1+o(1)], \quad h \rightarrow \infty .
$$

Note. A cruder formula

$$
\tau_{h}(x)=\tau(x)-\gamma \tau^{h}(x)[1+o(1)]
$$

follows directly from a general result due to Koenigs and Kneser (see Szekeres [20]).

PROOF OF LEMMA 3. Set $\tau_{h}=\tau-f_{n}, h \geq 0$; clearly $f_{0}=\tau-x \in(0, \tau)$. According to (1.1), (2.12),

$$
\begin{aligned}
f_{h+1} & =\tau-x \exp \left(\tau-f_{h}\right)=\tau-x e^{\tau} \exp \left(-f_{h}\right) \\
& =\tau\left[1-\exp \left(-f_{h}\right)\right], \quad h \geq 0,
\end{aligned}
$$

so, in particular, $f_{h} \in(0, \tau)$ for all $h \geq 0$. Subsequently,

$$
\left(f_{h} / \tau^{h}\right)\left[1-\exp \left(-f_{h}\right)\right] / f_{h} \leq f_{h+1} / \tau^{h+1} \leq f_{h} / \tau^{h}
$$

thus, by the right inequality, there exists $\gamma=\gamma(x)=\lim _{h \rightarrow \infty} f_{h} / \tau^{h}$ and, by the left inequality, $\gamma>0$ because

$$
\left[1-\exp \left(-f_{h}\right)\right] / f_{h}=1+O\left(f_{h}\right)=1+O\left(\tau^{h}\right)
$$

and $\tau<1$ for $x<e^{-1}$.

Set $f_{h}=\gamma \tau^{h}+g_{h}$; by definition of $\gamma, g_{h}=o\left(\tau^{h}\right)$ as $h \rightarrow \infty$. Using (2.15) again (bootstrapping!), after simple manipulations we get: for every $\varepsilon>0$ there exists $h(\varepsilon)$ such that, for $h \geq h(\varepsilon)$,

$$
\tau g_{h}-\left(\gamma^{2} \tau^{2 h+1} / 2\right)(1+\varepsilon) \leq g_{h+1} \leq \tau g_{h}-\left(\gamma^{2} \tau^{2 h+1} / 2\right)(1-\varepsilon) .
$$

Therefore, by induction, for $h^{\prime}>h \geq h(\varepsilon)$,

$$
\begin{aligned}
g_{h} / \tau^{h} & -\left[\gamma^{2} \tau^{h} / 2(1-\tau)\right]\left(1-\tau^{h^{\prime}-h}\right)(1+\varepsilon) \leq g_{h^{\prime}} / \tau^{h^{\prime}} \\
& \leq g_{h} / \tau^{h}-\left[\gamma^{2} \tau^{h} / 2(1-\tau)\right]\left(1-\tau^{h^{\prime}-h}\right)(1-\varepsilon) .
\end{aligned}
$$

Since $g_{h^{\prime}} / \tau^{h^{\prime}} \rightarrow 0$ as $h^{\prime} \rightarrow \infty$, the last double inequality implies that, for every $\varepsilon>0$

$$
g_{h}=\left[\gamma^{2} \tau^{2 h} / 2(1-\tau)\right](1+O(\varepsilon)), \quad h \rightarrow \infty
$$


This finishes the proof, because

$$
\tau_{h}(x)=\tau(x)-\gamma \tau^{h}(x)-g_{h}(x) .
$$

Using the estimate (2.14), we can now prove

LEMMA 4. For every $x \in\left(0, e^{-1}\right)$,

$$
T_{d}(x)=\left[\gamma^{2} \tau^{d-3}(x)(1-\tau(x)) / 2\right](1+o(1)), \quad d \rightarrow \infty .
$$

ProOF. (i) According to (2.13), for $\kappa>h$,

$$
\begin{aligned}
\sum_{j=2 h+1}^{2 \kappa+1} T_{j}^{0} & =\sum_{l=h}^{\kappa} \frac{\left(\tau_{l}-\tau_{l-1}\right)^{2}}{2}+\sum_{l=h}^{\kappa-1}\left[\left(\tau_{l+1}-\tau_{l}\right)-\tau_{l}\left(\tau_{l}-\tau_{l-1}\right)\right] \\
& =\tau_{\kappa}-\tau_{h}+\sum_{l=h}^{\kappa} \frac{\tau_{l-1}^{2}-\tau_{l}^{2}}{2}+\tau_{\kappa}\left(\tau_{\kappa}-\tau_{\kappa-1}\right) \\
& =\tau_{\kappa}-\tau_{h}+\left(\tau_{h-1}^{2}+\tau_{\kappa}^{2}\right) / 2-\tau_{\kappa} \tau_{\kappa-1}
\end{aligned}
$$

subsequently,

$$
T_{2 h+1}=\lim _{\kappa \rightarrow \infty} \sum_{j=2 h+1}^{2 \kappa+1} T_{j}^{0}=\tau-\tau_{h}+\frac{\tau_{h-1}^{2}-\tau^{2}}{2}
$$

Likewise,

$$
T_{2 h}=\tau-\tau_{h-1}-\left(\tau_{h-1}^{2}+\tau^{2}\right) / 2+\tau_{h-1} \tau_{h-2} .
$$

(ii) The relation (2.17) follows-after a straightforward computation-from Lemma 3 and (2.18), (2.19).

Now, back to the random graph $G(n, p)$.

LEMMA 5. Let $c \neq 1$. Given an integer $d$, denote the total number of the tree components of $G(n, p)$ of diameter $\geq d$ by $Y_{n}$. Define a number $y$ by

$$
d=(\log n+y) / \log (1 / \bar{\tau}),
$$

where $\bar{\tau} e^{-\bar{\tau}}=c e^{-c}$ and $\bar{\tau}<1$. If $d \rightarrow \infty$, when $n \rightarrow \infty$, in such a way that $y$ is bounded, then $Y_{n} \approx P\left(\lambda_{n}\right)$, where

$$
\lambda_{n}=\left[\bar{\gamma}^{2}(1-\bar{\tau}) / 2 c \bar{\tau}^{3}\right] e^{-y}, \quad \bar{\gamma}=\gamma\left(c e^{-c}\right) .
$$

Let us demonstrate how Theorem 2 follows from this lemma. If $c<1$, then the total number of vertices which belong to the nontree components of $G(n, p)$ is bounded in probability. Recalling that $\mathscr{P}_{n}$ is the length of the longest path in $G(n, p)$, we obtain then

$$
\begin{aligned}
P\left(\mathscr{P}_{n} \geq d\right) & =P\left(\mathscr{P}_{n} \geq d, Y_{n}>0\right)+P\left(\mathscr{P}_{n} \geq d, Y_{n}=0\right) \\
& =P\left(Y_{n}>0\right)+o(1) \\
& =1-\exp \left(-\lambda_{n}\right)+o(1), \quad \lambda_{n}=\left[\bar{\gamma}^{2}(1-c) / 2 c^{4}\right] e^{-y}
\end{aligned}
$$

$(\bar{\tau}=c$ for $c<1)$. 
ProOF OF Lemma 5. Similarly to (2.2),

$$
\begin{aligned}
E\left[\left(Y_{n}\right)_{r}\right]= & \sum\left(\begin{array}{c}
n \\
i_{1}, \ldots, i_{r}
\end{array}\right) \prod_{s=1}^{r} T\left(i_{s}, d\right) p^{i_{s}-1} q^{\left(i_{2}\right)-\left(i_{s}-1\right)+\left(n-i_{s}\right) i_{s}} \\
& \cdot \prod_{1 \leq s \neq s^{\prime} \leq r} q^{-i_{s} i_{s^{\prime}} / 2},
\end{aligned}
$$

where $i_{s}>d, 1 \leq s \leq r$. (A tree with $i$ vertices has $(i-1)$ edges.) Let again $\Sigma^{\prime}, \Sigma^{\prime \prime}$ be the partial sums of the sum in (2.20) over the regions $\max _{s} i_{s} \leq n_{1}$ and $\max _{s} i_{s}>n_{1}$ respectively. Then (cf. (2.4)) we have

$$
\Sigma^{\prime}=\left[\frac{n}{c} \sum_{i=d+1}^{n_{1}} \frac{x^{i} T(i, d)}{i !}\right]^{r}\left(1+O\left(n^{-1 / 3}\right)\right), \quad x=c e^{-c} .
$$

Using Lemma 4, an obvious estimate $T(i, d) \leq t(i)<i^{i}$, and also that $e c e^{-c}<1$, we write

$$
\begin{aligned}
\frac{n}{c} \sum_{i=d+1}^{n_{1}} \frac{x^{i} T(i, d)}{i !} & =\left(\frac{n}{c}\right) T_{d}(x)+O\left(n \sum_{i>n_{1}} \frac{x^{i} i^{i}}{i !}\right) \\
& =\left[(n / c) \bar{\gamma}^{2} \bar{\tau}^{d-3}(1-\bar{\tau}) / 2\right](1+o(1))+o(1)=\lambda_{n}+o(1),
\end{aligned}
$$

whence

$$
\left.\Sigma^{\prime}=\lambda_{n}^{r}(1+o(1))\right) .
$$

As for $\Sigma^{\prime \prime}$, comparing (2.2) and (2.20) yields an estimate $\Sigma^{\prime \prime}=O\left(n^{r} \widetilde{\Sigma}^{\prime \prime}\right)$, where $\widetilde{\Sigma}^{\prime \prime}$ is defined by (2.6). Therefore, see (2.9),

$$
\Sigma^{\prime \prime}=O\left(n^{2 r} \rho_{1}^{n_{1}}\right)=o(1), \quad n \rightarrow \infty .
$$

Thus,

$$
E\left[\left(Y_{n}\right)_{r}\right]=\Sigma^{\prime}+\Sigma^{\prime \prime}=\lambda_{n}^{r}(1+o(1)), \quad r \geq 1,
$$

which implies the statement.

Theorem 2 is proven.

Now, contrary to the listing of the theorem in Introduction, we proceed with the proof of Theorem 3, since- by methods used-it is closely related to the proofs above.

Let $U_{n s}$ and $V_{n s}$ denote the total number of unicyclic components of order $=s$, and the total number of unicyclic components whose cycle is of length $=s, s \geq 3$. Obviously,

$$
\mathscr{U}_{n}=\sum_{s=3}^{n} s U_{n s}, \quad \mathscr{V}_{n}=\sum_{s=3}^{n} s V_{n s} .
$$

Erdős and Rényi [9] proved that, for $c \in(0, \infty)$,

$$
E\left(\left(U_{n s}\right)_{\kappa}\right) \rightarrow \mu_{s}^{\kappa}, \quad \kappa \geq 1, s \geq 3,
$$

where

$$
\mu_{s}=\left(c e^{-c}\right)^{s} u(s) / s !, \quad s \geq 3,
$$


which implies that $U_{n s} \Rightarrow P\left(\mu_{s}\right)$. (To be precise, they dealt with the random graph $G(n, M)$.) By computing the joint factorial moments of $U_{n s}, s \geq 3$, Bollobás [6] proved that actually

$$
\left(U_{n 3}, \ldots, U_{n \kappa}\right) \Rightarrow\left(P\left(\mu_{3}\right), \ldots, P\left(\mu_{\kappa}\right)\right), \quad \kappa \geq 3,
$$

where the Poisson variables are independent. By a natural extension of the argument in the proof of Lemma 2, it is possible to estimate the joint factorial moments of $V_{n s}, s \geq 3$, as well. The result is that, for $c \neq 1$,

$$
\left(V_{n 3}, \ldots, V_{n \kappa}\right) \Rightarrow\left(P\left(\nu_{3}\right), \ldots, P\left(\nu_{\kappa}\right)\right), \quad \kappa \geq 3,
$$

where

$$
\nu_{s}=u_{s}^{0}\left(c e^{-c}\right)=\sum_{r \geq s} \frac{\left(c e^{-c}\right)^{r} u^{0}(r, s)}{r !}, \quad s \geq 3,
$$

and $u^{0}(r, s)$ is the total number of all connected unicyclic graphs with a cycle of length $=s$. Let us notice at once that, see Lemma 1 ,

$$
\nu_{s}=u_{s}^{0}\left(c e^{-c}\right)=u_{s-1}\left(c e^{-c}\right)-u_{s}\left(c e^{-c}\right)=\tau^{s}\left(c e^{-c}\right) / 2 s, \quad s \geq 3,
$$

(cf. [9, Theorem 3a; 6, Chapter IV, Corollary 9]). Therefore,

$$
\mathscr{U}_{n}(\kappa) \underset{\text { def }}{=} \sum_{s=3}^{\kappa} s U_{n s} \Rightarrow \mathscr{U}(\kappa), \quad \mathscr{V}_{n}(\kappa) \underset{\text { def }}{=} \sum_{s=3}^{\kappa} s V_{n s} \Rightarrow \mathscr{V}(\kappa)
$$

where the random variables $\mathscr{U}(\kappa)$ and $\mathscr{V}(\kappa)$ are such that

$$
\begin{gathered}
E\left(z^{\mathscr{U}(\kappa)}\right)=\exp \left[\sum_{s=3}^{\kappa} \frac{\left(z c e^{-c}\right)^{s} u(s)}{s !}-\sum_{s=3}^{\kappa} \frac{\left(c e^{-c}\right)^{s} u(s)}{s !}\right], \\
E\left(z^{\mathscr{V}(\kappa)}\right)=\exp \left\{\sum_{s=3}^{\kappa} \frac{\left[z \tau\left(c e^{-c}\right)\right]^{s}}{2 s}-\sum_{s=3}^{\kappa} \frac{\tau^{s}\left(c e^{-c}\right)}{2 s}\right\} .
\end{gathered}
$$

Suppose that $c \neq 1$. Then $c e^{-c}<e^{-1}$, and it follows from (2.1) and (2.22), (2.23) that, for $|z|<\left(e c e^{-c}\right)^{-1}$,

$$
\begin{gathered}
\lim _{\kappa \rightarrow \infty} E\left(z^{\mathscr{U}(\kappa)}\right)=f(\bar{\tau}) / f\left[\tau\left(z c e^{-c}\right)\right], \\
\lim _{\kappa \rightarrow \infty} E\left(z^{\mathscr{V}(\kappa)}\right)=f(\bar{\tau}) / f(z \bar{\tau}), \quad \bar{\tau}=\tau\left(c e^{-c}\right),
\end{gathered}
$$

see (1.9) for $f(\cdot)$.

We want to show that, for $|z|=1$,

$$
\begin{gathered}
\lim _{n \rightarrow \infty} E\left(z^{\mathscr{U _ { n }}}\right)=f(\bar{\tau}) / f\left[\tau\left(z c e^{-c}\right)\right] \underset{\mathrm{def}}{=} E\left(z^{\mathscr{U}}\right), \\
\lim _{n \rightarrow \infty} E\left(z^{\mathscr{V}_{n}}\right)=f(\bar{\tau}) / f(z \bar{\tau}) \underset{\text { def }}{=} E\left(z^{\mathscr{V}}\right) .
\end{gathered}
$$

Consider (2.27), for instance. Since $E\left(\mathscr{V}_{n}\right)=O(1)$, see (2.32) in the sequel,

$$
P\left(\mathscr{V}_{n}(\kappa) \neq \mathscr{V}_{n}\right) \leq P\left(\mathscr{V}_{n}>\kappa\right) \leq E\left(\mathscr{V}_{n}\right) / \kappa=O\left(\kappa^{-1}\right), \quad n \geq 1 .
$$

Further,

$$
\begin{aligned}
\left|E\left(z^{\mathscr{V} n}\right)-f(\bar{\tau}) / f(z \bar{\tau})\right| \leq & \left|E\left(z^{\mathscr{V} n}-z^{\mathscr{V}_{n}(\kappa)}\right)\right|+\left|E\left(z^{\mathscr{\mathscr { V } _ { n }}(\kappa)}\right)-E\left(z^{\mathscr{V}(\kappa)}\right)\right| \\
+ & \left|E\left(z^{\mathscr{V}(\kappa)}\right)-f(\bar{\tau}) / f(z \bar{\tau})\right|,
\end{aligned}
$$


where, by $(2.28)$,

$$
\left|E\left(z^{\mathscr{V}_{n}}-z^{\mathscr{V}_{n}(\kappa)}\right)\right| \leq 2 P\left(\mathscr{V}_{n}(\kappa) \neq \mathscr{V}_{n}\right)=O\left(\kappa^{-1}\right) .
$$

So, given $\varepsilon>0$, we can choose $\kappa$ so large that the sum of the first and the last terms on the right side of (2.29) is less than $\varepsilon$. Letting $n \rightarrow \infty$, we get then

$$
\limsup _{n}\left|E\left(z^{\mathscr{V}_{n}}\right)-f(\bar{\tau}) / f(z \bar{\tau})\right| \leq \varepsilon, \quad \forall \varepsilon>0 .
$$

So (2.27) holds true.

In view of (2.26) and (2.27), we conclude that $\mathscr{U}_{n} \Rightarrow \mathscr{U}, \mathscr{V}_{n} \Rightarrow \mathscr{V}$.

Finally, to show that actually $\mathscr{U}_{n} \stackrel{m}{\Rightarrow} \mathscr{U}, \mathscr{V}_{n} \stackrel{m}{\Rightarrow} \mathscr{V}$, we need only to prove

$$
E\left(\mathscr{U}_{n}^{\kappa}\right)=O(1), \quad E\left(\mathscr{V}_{n}^{\kappa}\right)=O(1), \quad n \rightarrow \infty, \kappa \geq 1,
$$

and it suffices to consider the moments of $\mathscr{U}_{n}$, because $\mathscr{U}_{n} \geq \mathscr{V}_{n}$.

First of all,

$$
E\left(\left(\mathscr{U}_{n}\right)_{\kappa}\right)=(n)_{\kappa} P_{n \kappa},
$$

where $P_{n \kappa}$ is the probability that some fixed $\kappa$ vertices, say $1,2, \ldots, \kappa$, belong to the unicyclic components of $G(n, p)$. How to compute $P_{n \kappa}$ ? For a given partition $\mathscr{R}$ of $\{1, \ldots, \kappa\}$ into the disjoint subsets $R_{1}, \ldots, R_{l}$, let $P_{n}(\mathscr{R})$ denote the probability that the elements of each $R_{j}, 1 \leq j \leq l$, belong to the same unicyclic component; then

$$
P_{n \kappa}=\sum_{\mathscr{R}} P_{n}(\mathscr{R}) .
$$

As for $P_{n}(\mathscr{R})$, denoting $\left|R_{s}\right|$ by $a_{s}, 1 \leq s \leq r$, we have

$$
\begin{aligned}
& P_{n}(\mathscr{R})=\sum_{j_{1}, \cdots, j_{r}}\left(\begin{array}{c}
n-\kappa \\
j_{1} \ldots j_{r}
\end{array}\right) \prod_{s=1}^{r} u\left(i_{s}\right) p^{i_{s}} q^{\left(i_{2} s\right)-i_{s}+\left(n-i_{s}\right) i_{s}} \prod_{1 \leq s \neq s^{\prime} \leq r} q^{-i_{s} i_{s^{\prime}} / 2}, \\
& i_{s}=j_{s}+a_{s} .
\end{aligned}
$$

So, arguing as in the proof of Lemma 2, we get

$$
\begin{aligned}
P_{n}(\mathscr{R}) & =\left(1+O\left(n^{-1 / 3}\right)\right) \prod_{s=1}^{r} \sum_{j_{s} \geq 0}\left(\frac{n^{j_{s}}}{j_{s} !}\right) u\left(i_{s}\right) p^{i_{s}} e^{-c i_{s}} \\
& =\left(1+O\left(n^{-1 / 3}\right)\right) n^{-\kappa} \prod_{s=1}^{r}\left[\sum_{i \geq a_{s}} \frac{\left(c e^{-c}\right)^{i} u(i)}{\left(i-a_{s}\right) !}\right] .
\end{aligned}
$$

Therefore, by (2.31),

$$
\lim _{n \rightarrow \infty} E\left(\left(\mathscr{U}_{n}\right)_{\kappa}\right)=\sum_{\mathscr{R}} \prod_{s=1}^{r}\left[\sum_{i \geq a_{s}} \frac{\left(c e^{-c}\right)^{i} u(i)}{\left(i-a_{s}\right) !}\right]<\infty .
$$

This, of course, implies (2.30), since

$$
\mathscr{U}_{n}^{\kappa}=\sum_{j=0}^{\kappa} \sigma(\kappa, j)\left(\mathscr{U}_{n}\right)_{j}
$$

where $\sigma(\kappa, j)$ are the Stirling numbers of the second kind, Berge [3]. 
Theorem 3 follows now from (2.26), (2.27) and (2.30), because $\bar{\tau}=c$ for $c<1$.

Note. The relations (2.26), (2.27) could have also been obtained by algebraic arguments directly from (2.32), and a similar formula for $\lim E\left(\left(\mathscr{V}_{n}\right)_{\kappa}\right)$. Would the reader try to do it? We have chosen the present derivation as far more transparent probabilistically.

To prove the remaining theorems, we need a more powerful tool.

For two given sets of nonnegative integers $j=\left\{j_{r}: 1 \leq r \leq n\right\}$ and $\kappa=$ $\left\{\kappa_{s}: 3 \leq s \leq n\right\}$ satisfying

$$
\sum_{r} r j_{r}+\sum_{s} s \kappa_{s}=n
$$

introduce the event $A_{n}(j, \kappa)$ on which the graph $G(n, p)$ has $j_{r}$ tree-components of size $r(1 \leq r \leq n)$, and $\kappa_{s}$ unicyclic components of size $s(3 \leq s \leq n)$. (On this event, $G(n, p)$ has obviously no components besides those listed above.) Denoting the probability of $A_{n}(j, \kappa)$ by $P_{n}(j, \kappa)$, we have

$$
\begin{aligned}
P_{n}(j, \kappa)= & n ! \prod_{r}\left[p^{r-1} q^{\left(\begin{array}{c}
r \\
2
\end{array}\right)-(r-1)} t_{*}(r) / r !\right]^{j_{r}} / j_{r} ! \prod_{s}\left[p^{s} q_{s}^{\left(\begin{array}{l}
(s) \\
2
\end{array}\right)} u(s) / s !\right]^{\kappa_{s}} / \kappa_{s} ! \\
& \cdot q^{n^{2} / 2} \prod_{r}\left(q^{-r^{2} / 2}\right)^{j_{r}} \prod_{s}\left(q^{-s^{2} / 2}\right)^{\kappa_{s}},
\end{aligned}
$$

where $t_{*}(r)=r^{r-2}$ is the total number of unrooted trees on $r$ vertices, and $u(s)$ is the total number of connected unicyclic graphs on $s$ vertices. Indeed,

$$
n ! / \prod_{r}(r !)^{j_{r}} j_{r} ! \cdot \prod_{s}(s !)^{\kappa_{s}} \kappa_{s} !
$$

is the total number of ways to partition the set of $n$ vertices into the blocks of two types, so that there are $j_{r}$ first-type blocks of size $r$ and $\kappa_{s}$ second-type blocks of size $s(1 \leq r \leq n, 3 \leq s \leq n)$. Further, the product of all the remaining factors in (2.34) is the probability that each of the correspondent subgraphs of $G(n, p)$ is either a tree-component, if it is induced by a first-type block, or a unicyclic component otherwise. (Cf. the derivativon of (2.2).) Combining factors and using (2.23), we can simplify (2.34) to

$$
P_{n}(j, \kappa)=\omega_{n} \prod_{r}\left[(q / p) t_{*}(r) / r !\right]^{j_{r}} / j_{r} ! \prod_{s}[u(s) / s !]^{\kappa_{s}} / \kappa_{s} !
$$

where

$$
\omega_{n}=n ! p^{n} q^{n(n-3) / 2} .
$$

Note. Not too surprisingly perhaps, there is a strong semblance between (2.35) and the counting formulae in the graph and partition-type problems, Berge [3], Harary and Palmer [10].

Basically, two reasons make (2.35) instrumental in the arguments below. First, for $c<1$-which is assumed in the rest of the paper-the components of $G(n, p)$ are either trees or unicyclic with probability $\geq 1-$ const $n^{-w}(\forall w<1)$, whence

$$
\sum_{j, \kappa} P_{n}(j, \kappa)-1=O\left(n^{-w}\right), \quad \forall w<1 .
$$


Second, we know the exponential generating functions $u(x)$ of $\{u(s): s \geq 3\}$, see (2.1), and $\tau_{*}(x)$ of $\left\{t_{*}(r): r \geq 1\right\}$ (Moon [12]):

$$
\tau_{*}(x)=\tau(x)-\tau^{2}(x) / 2, \quad|x|<e^{-1} .
$$

After these preparations, we are ready for

PROOF OF THEOREM 3.

LEMMA 6. Suppose that $c<1$. For a given integer $m$, introduce the random variable $Z_{n m}$ which is the total number of the tree-components of order $\geq m$. Denote $E\left(Z_{n m}\right)$ by $\lambda_{n m}$. If $m \rightarrow \infty, \lambda_{n m} \rightarrow \infty$ when $n \rightarrow \infty$, then $\left(Z_{n m}-\lambda_{n m}\right) / \lambda_{n m}^{1 / 2} \Rightarrow Z$, where $Z$ is normal with 0 mean and unit variance.

Notes. (1) Let $Z_{n m}^{0}$ denote the total number of the tree components of order $=m$. Erdős and Rényi [9] proved, for $c \neq 1$, that $Z_{n m} \approx P\left(\lambda_{n m}\right), Z_{n m}^{0} \approx$ $P\left(\lambda_{n m}^{0}\right)\left(\lambda_{n m}=E\left(Z_{n m}^{0}\right)\right)$, provided that $m \rightarrow \infty$ so fast that $\lambda_{n m}$-and automatically $\lambda_{n m}^{0}$-remains bounded. (This happens iff

$$
m=\alpha^{-1}\left(\log n-\frac{5}{2} \log \log n\right)+O(1), \quad n \rightarrow \infty .
$$

Therefore, under conditions of Lemma 6 ,

$$
m<\alpha^{-1} \log n
$$

for large enough $n$.)

(2) From a general theorem due to Barbour [2], it follows that, in terms of the total variation, the distribution of $Z_{n m}^{0}$ is close to that of $P\left(\lambda_{n m}^{0}\right)$ provided only that $m \rightarrow \infty$. In light of these results, we see that our assertion could be anticipated: after all, $P(\lambda)$ is asymptotically normal, with mean and variance both equal $\lambda$ when $\lambda \rightarrow \infty$. However, the argument we suggest below works only for $c<1$, and we can only conjecture that the statement remains true for $c>1$ as well.

PROOF OF LEMMA 6 . Let $z=\exp \left(i v / \lambda_{n m}^{1 / 2}\right), v \in(-\infty, \infty)$. Since $Z_{n m}=$ $\sum_{r=m}^{n} j_{r}$ on the event $A_{n}(j, \kappa)$, we have (see (2.35) and (2.37))

$$
\begin{aligned}
E\left(z^{Z_{n m}}\right) & =o(1)+\omega_{n} \sum_{j, \kappa} \prod_{r=1}^{n}\left[(q / p) z \delta(r) t_{*}(r) / r !\right]^{j_{r}} / j_{r} ! \cdot \prod_{s=1}^{n}[u(s) / s !]^{\kappa_{s}} / \kappa_{s} ! \\
& =o(1)+\omega_{n} R_{n m}(z),
\end{aligned}
$$

where $\delta(r)=0(\delta(r)=1)$ for $r<m(r \geq m)$. Let $|x|<e^{-1}$; using (2.33) we are led to

$$
\begin{aligned}
1+\sum_{n \geq 1} x^{n} R_{n m}(z) & =1+\sum_{n \geq 1} \sum_{j, \kappa} \prod_{r=1}^{n} \frac{\left[(q / p) z x^{r} \delta(r) t_{*}(r) / r !\right]^{j_{r}}}{j_{r} !} \cdot \prod_{s=1}^{n} \frac{\left[x^{s} u(s) / s !\right]^{\kappa_{s}}}{\kappa_{s} !} \\
& =1+\prod_{1 \leq 1\left(j_{1}+\kappa_{1}\right)+2\left(j_{2}+\kappa_{2}\right) \cdots<\infty} \frac{[]_{r}^{j_{r}}}{j_{r} !} \cdot \prod_{s \geq 1} \frac{[]^{\kappa s}}{\kappa_{s} !} \\
& =\prod_{r \geq 1} \exp \left[\frac{(q / p) z x^{r} \delta(r) t_{*}(r)}{r !}\right] \prod_{s \geq 1} \exp \left[\frac{x^{s} u(s)}{s !}\right] \\
& =\exp \left[\left(\frac{q}{p}\right) z \sum_{r \geq 1} \frac{x^{r} \delta(r) t_{*}(r)}{r !}+u(x)\right] \\
& =\exp [h(x, z)] .
\end{aligned}
$$


By Cauchy integral formula, it follows from (2.41) that, for a positively oriented circle of radius $\rho<e^{-1}$ in the $x$-plane,

$$
\begin{aligned}
R_{n m}(z) & =(2 \pi i)^{-1} \int_{C} x^{-(n+1)} \exp [h(x, z)] d x \\
& =(2 \pi)^{-1} \int_{C} \exp [H(x, z)](i x)^{-1} d x
\end{aligned}
$$

here, (see (2.38) and (2.41)),

$$
\begin{aligned}
H(x, z) & =\left(\frac{q}{p}\right) \tau_{*}(x)+\frac{q}{p}(z-1) \sum_{r \geq m} x^{r} t_{*}(r) / r !+u(x)-n \log x \\
& =\left(\frac{q}{p}\right)\left[\tau(x)-\frac{\tau^{2}(x)}{2}\right]+\left(\frac{q}{p}\right)(z-1) \sum_{r \geq m} \frac{x^{r} r^{r-2}}{r !}+u(x)-n \log x
\end{aligned}
$$

and $\log x$ is the principal branch of the logarithmic function.

Let us use the saddle-point method to estimate the contour integral in (2.42). To this purpose, set $\rho=\rho_{n}=\left|x_{n}\right|$ where $x_{n}$ is a root of the equation $H_{x}(x, z)=0$ in the domain $|x|<e^{-1}$. According to (1.1), (2.1),

$$
\tau_{x}=e^{\tau} /(1-\tau), \quad u_{x}=\tau^{2} e^{\tau} / 2(1-\tau)^{2},
$$

so explicitly

$$
\begin{aligned}
H_{x}(x, z)= & c^{-1}(n-c) e^{\tau}-n / x \\
& +c^{-1}(n-c)(z-1) \sum_{r \geq m} \frac{x^{r-1} r^{r-1}}{r !}+\frac{\tau^{2} e^{\tau}}{2(1-\tau)^{2}}=0 .
\end{aligned}
$$

Since $z-1 \rightarrow 0, n \rightarrow \infty$ and $\sum_{r \geq m} x^{r-1} r^{r-1} / r$ ! is bounded-uniformly over $m \geq 1$-in every closed disk $|x| \leq \rho\left(\rho<e^{-1}\right)$, it is natural to look first for a root of an approximate equation

$$
c^{-1} e^{\tau(x)}-x^{-1}=0 \quad\left(|x|<e^{-1}\right) .
$$

But this equation does have a root $\bar{x}=c e^{-c}$ because $c<1$ ! (Recall that $\tau\left(c e^{-c}\right)=c$ for $c<1$.) Further,

$$
\left.\left(c^{-1} e^{\tau(x)}-x^{-1}\right)^{\prime}\right|_{x=\bar{x}} \neq 0
$$

so, with the help of the implicit function theorem, we get: there exists a root $x_{n}$ of (2.44) such that

$$
x_{n}-\bar{x}=o(1), \quad n \rightarrow \infty .
$$

Since $\rho_{n}=\left|x_{n}\right|<e^{-1}$ and $\rho_{n}$ is bounded away from $e^{-1}$, we can obtain from (2.44)

$$
c^{-1} e^{\tau\left(x_{n}\right)}-x_{n}^{-1}=O\left(\lambda_{n m}^{-1 / 2} \rho_{n}^{m} m^{m-1} / m !+n^{-1}\right),
$$

or, by (2.46),

$$
x_{n}-\bar{x}=O\left(\lambda_{n m}^{-1 / 2} \rho_{n}^{m} m^{m-1} / m !+n^{-1}\right) .
$$

Some easy bootstrapping transforms (2.47) into

$$
x_{n}-\bar{x}=O\left(\lambda_{n m}^{-1 / 2} \bar{x}^{m} m^{m-1} / m !+n^{-1}\right) .
$$


To simplify the last estimate further, notice that

$$
\lambda_{n m}=\sum_{j \geq 1}\left(\begin{array}{l}
n \\
j
\end{array}\right) j^{j-2} p^{j-1} q^{\left(\begin{array}{l}
j \\
2
\end{array}\right)-(j-1)+j(n-j)} .
$$

So, acting as in the proof of Lemma 2, but with $n_{1}=\left[n^{1 / 5}\right]$, and introducing

$$
a_{n m}=c^{-1} n \sum_{j \geq m} \frac{\bar{x}^{j} j^{j-2}}{j !}
$$

we have

$$
\lambda_{n m}=\left(1+O\left(n^{-3 / 5}\right)\right) a_{n m}=a_{n m}+o\left(a_{n m}^{1 / 2}\right)=a_{n m}+o\left(\lambda_{n m}^{1 / 2}\right),
$$

because $\lambda_{n m} \rightarrow \infty, \lambda_{n m} \leq n$. Also, by Stirling's formula,

$$
\begin{aligned}
a_{n m} & =(1+o(1)) c^{-1} n \sum_{j \geq m} \frac{(e \bar{x})^{j}}{(2 \pi)^{1 / 2} j^{5 / 2}} \\
& =(1+o(1)) n\left(\sigma e^{\alpha m} m^{5 / 2}\right)^{-1}, \\
\alpha & =c-1-\log c, \quad \sigma=(2 \pi)^{1 / 2} c\left(1-e^{-\alpha}\right),
\end{aligned}
$$

(cf. [9]). A combination of (2.48)-(2.51) yields

$$
x_{n}-\bar{x}=O\left(m \lambda_{n m}^{1 / 2} / n\right)=o\left(n^{-1 / 2} e^{-\alpha m / 2}\right),
$$

or, writing $x_{n}=\rho_{n} e^{i \theta_{n}}, \theta_{n} \in(-\pi, \pi]$,

$$
\left|\rho_{n}-\bar{x}\right|,\left|\theta_{n}\right|=o\left(n^{-1 / 2} e^{-\alpha m / 2}\right) .
$$

Getting back to (2.42), let us substitute $x=\rho_{n} e^{i \theta}, \theta \in(-\pi, \pi]$, and break the contour integral into two integrals over the $\operatorname{arcs} C_{1}$ and $C_{2}$, such that $|\theta| \leq n^{-5 / 12}$ and $|\theta|>n^{-5 / 12}$ respectively.

(a) A direct computation based on (2.50)-(2.53) shows that

$$
H_{x x}(x, z)=n / \bar{x}^{2}(1-c)+O\left(n^{7 / 12}\right), \quad|\theta| \leq n^{-5 / 12} .
$$

So, for these $\theta$ 's, by (2.44),

$$
\begin{aligned}
H(x, z) & =H\left(x_{n}, z\right)+H_{x}\left(x_{n}, z\right)\left(x-x_{n}\right)+H_{x x}(\tilde{x}, z)\left(x-x_{n}\right)^{2} / 2 \\
& =H\left(x_{n}, z\right)-[n /(1-c)]\left(\theta-\theta_{n}\right)^{2} / 2+O\left(n^{-1 / 4}\right),
\end{aligned}
$$

where $\tilde{x}=\rho_{n} e^{i \tilde{\theta}},|\tilde{\theta}| \leq n^{-5 / 12}$. Furthermore, by (2.45) and (2.52), we have

$$
\begin{aligned}
H\left(x_{n}, z\right) & =H(\bar{x}, z)+H_{x}(\bar{x}, z)\left(x_{n}-\bar{x}\right)+O\left(n\left|x_{n}-\bar{x}\right|^{2}\right) \\
& =H(\bar{x}, z)+O\left(m^{2} \lambda_{n m} / n\right)=H(\bar{x}, z)+o\left(e^{-\alpha m}\right) .
\end{aligned}
$$

Likewise,

$$
H\left(\rho_{n}, z\right)=H(\bar{x}, z)+o\left(e^{-\alpha m}\right),
$$

which will be needed later. By (2.54) and (2.55),

$$
\begin{aligned}
\int_{C_{1}}= & \exp \left[H(\bar{x}, z)+o\left(e^{-\alpha m}\right)\right] \\
& \cdot \int_{|\theta| \leq n^{-5 / 12}} \exp \left[-n\left(\theta-\theta_{n}\right)^{2} / 2(1-c)+O\left(n^{-1 / 4}\right)\right] d \theta \\
= & \exp [H(\bar{x}, z)][2 \pi(1-c) / n]^{1 / 2}(1+o(1)), \quad n \rightarrow \infty .
\end{aligned}
$$


(b) Turn now to $|\theta|>n^{-5 / 12}$. We have, see (2.43) and (2.56),

$$
\begin{aligned}
& \operatorname{Re}[H(x, z)-H(\bar{x}, z)]=\operatorname{Re}\left[H(x, z)-H\left(\rho_{n}, z\right)\right]+o\left(e^{-\alpha m}\right) \\
& =(q / p) \operatorname{Re}\left[\tau_{*}(x)-\tau_{*}\left(\rho_{n}\right)\right] \\
& \quad+O\left(n|z-1| \sum_{r \geq m} \frac{\rho_{n}^{r} r^{r-2}}{r !}\left|e^{i \theta r}-1\right|\right)+o\left(e^{-\alpha m}\right) .
\end{aligned}
$$

Here

$$
(q / p) \operatorname{Re}\left[\tau_{*}(x)-\tau_{*}\left(\rho_{n}\right)\right] \leq(q / p) \rho_{n}(\cos \theta-1) \leq-\chi_{1} n \theta^{2} \leq-\chi_{1} n^{1 / 6}
$$

and $\left(\left|e^{i \theta r}-1\right| \leq r|\theta|\right)$

$$
\begin{aligned}
n \mid z- & 1\left|\sum_{r \geq m} \frac{\rho_{n}^{r} r^{r-2}}{r !}\right| e^{i \theta r}-1|=n| z-1|| \theta \mid \sum_{r \geq m} \frac{\rho_{n}^{r} r^{r-1}}{r !} \\
= & O\left(n|z-1||\theta| \sum_{r \geq m} \frac{\bar{x}^{r} r^{r-1}}{r !}\right)=O\left(m \lambda_{n m}^{1 / 2}|\theta|\right)=o\left(n^{1 / 2}|\theta|\right) .
\end{aligned}
$$

But

$$
n \theta^{2} / n^{1 / 2}|\theta|=n^{1 / 2} \theta \geq n^{1 / 12} \rightarrow \infty \quad\left(|\theta|>n^{-5 / 12}\right)
$$

so

$$
\operatorname{Re}[H(x, z)-H(\bar{x}, z)] \leq-\chi_{2} n^{1 / 6}, \quad \chi_{1}>\chi_{2}>0 .
$$

Subsequently,

$$
\left|\int_{C_{2}}\right|=O\left(\left|\exp [H(\bar{x}, z)] \exp \left(-\chi_{2} n^{1 / 6}\right)\right|\right)=o\left(\left|\int_{C_{1}}\right|\right),
$$

see (2.57).

In view of $(2.40),(2.57)$ and $(2.58)$, we arrive at

$$
E\left(z^{Z_{n m}}\right)=o(1)+\omega_{n}(2 \pi)^{-1}[2 \pi(1-c) / n]^{1 / 2} \exp [H(\bar{x}, z)](1+o(1)) .
$$

In this relation, by (2.36) and Stirling's formula for $n$ !,

$$
\omega_{n}=(2 \pi n)^{1 / 2}(c / e)^{n} \exp \left(-c n / 2+3 c / 2-c^{2} / 4+O\left(n^{-1}\right)\right) .
$$

Furthermore, by (2.1), (2.38),

(2.61) $H(\bar{x}, z)=(q / p)\left(c-c^{2} / 2\right)-n \log \left(c e^{-c}\right)+\log (1-c)^{-1 / 2}-c / 2-c^{2} / 4+\Delta H$, where $\left(z=\exp \left(i v / \lambda_{n m}^{1 / 2}\right)\right)$

$$
\begin{aligned}
\Delta H & =\left(\frac{q}{p}\right)(z-1) \sum_{r \geq m} \frac{\bar{x}^{r} r^{r-2}}{r !} \\
& =(1-c / n) a_{n m}\left[\exp \left(i v / \lambda_{n m}^{1 / 2}\right)-1\right] \\
& =a_{n m}\left[i v / \lambda_{n m}^{1 / 2}-v^{2} / 2 \lambda_{n m}+O\left(\lambda_{n m}^{-3 / 2}\right)\right]+O\left(a_{n m} / n\right) \\
& =i v\left[\lambda_{n m}^{1 / 2}+o(1)\right]-v^{2} / 2+o(1),
\end{aligned}
$$

(see (2.49)-(2.51)). Putting together the relations (2.59)-(2.62), we are able to conclude, after numerous cancellations, that, for every $v \in(-\infty, \infty)$,

$$
E\left[\exp \left(i v Z_{n m} / \lambda_{n m}^{1 / 2}\right)\right]=o(1)+\exp \left(i v \lambda_{n m}^{1 / 2}-v^{2} / 2+o(1)\right), \quad n \rightarrow \infty .
$$


This implies that $\left(Z_{n m}-\lambda_{n m}\right) / \lambda_{n m}^{1 / 2}$ converges in distribution to a standard normal variable.

With the help of this lemma, we can now quickly finish the proof of Theorem 3. So, suppose $\nu \rightarrow \infty, \nu=o\left(n / \log ^{5 / 2} n\right)$, and set

$$
s_{n \nu}=\alpha^{-1} \log \left(n \delta / \nu \log ^{5 / 2} n\right), \quad \delta=\alpha^{5 / 2} \sigma^{-1},
$$

(see $(2.51)$ for $\sigma$ ). It is sufficient to show that $\hat{\mathscr{S}}_{n \nu}$, the order of the $\nu$ th largest tree-component a.s. equals either $\left\langle s_{n \nu}\right\rangle$, or $\left\langle s_{n \nu}\right\rangle-1$; recall that $\left\langle s_{n \nu}\right\rangle$ is an integer closest to $s_{n \nu}$.

Introduce $m_{1}=\left\langle s_{n \nu}\right\rangle+1$, so that $\Delta=m_{1}-s_{n \nu} \geq 1 / 2$. Obviously, $m_{1} \rightarrow \infty$ and, by (2.50) and (2.51),

$$
\lambda_{n m_{1}}=(1+o(1)) \nu e^{-\alpha \Delta} \leq \nu e^{-\alpha / 3} .
$$

Therefore,

$$
P\left(\hat{\mathscr{S}}_{n \nu} \geq m_{1}\right)=P\left(Z_{n m_{1}} \geq \nu\right) \leq P\left(Z_{n m_{1}} \geq e^{\alpha / 3} \lambda_{n m_{1}}\right) \rightarrow 0, \quad n \rightarrow \infty,
$$

since, by Lemma $6, Z_{n m_{1}} / \lambda_{n m_{1}} \rightarrow 1$ in probability. Similarly, setting $m_{2}=$ $\left\langle s_{n \nu}\right\rangle-1$, we have $\Delta=m_{2}-s_{n \nu} \leq-1 / 2$; so

$$
\lambda_{n m_{2}}=(1+o(1)) \nu e^{-\alpha \Delta} \geq \nu e^{\alpha / 3}
$$

and

$$
P\left(\hat{\mathscr{S}}_{n \nu} \geq m_{2}\right)=P\left(Z_{n m_{2}} \geq \nu\right) \geq P\left(Z_{n m_{2}} \geq e^{-\alpha / 3} \lambda_{n m_{2}}\right) \rightarrow 1, \quad n \rightarrow \infty .
$$

Theorem 3 is proven. Finally,

PROOF OF THEOREM 4. We confine ourselves to proving only that

$$
\mathscr{D}_{n}^{\prime}-D_{n} \approx \mathscr{E}_{n}^{\prime}, \quad n \rightarrow \infty,
$$

since the proof of (1.6) is very much the same, albeit rather cumbersome notationally.

According to (1.7), $\log E\left(z^{\mathscr{E}_{n}^{\prime}}\right)$ is bounded as $n \rightarrow \infty$ for every $z \in(0, \infty)$. Using a limit theorem due to Curtiss [7], we can assert then that (2.63) will be proved if we demonstrate that

$$
E\left(z^{\mathscr{D}_{n}^{\prime}-D_{n}}\right)-E\left(z^{\mathscr{E}_{n}^{\prime}}\right) \rightarrow 0, \quad n \rightarrow \infty
$$

for every $z \in\left(a, a^{-1}\right)$, where $0<a<1$. A little reflection shows that, more generally, it would suffice to prove (2.64) for every $z \in(a, b)$, where $0<a<b$.

LEMMA 7. For a given $m$, let $\mathscr{D}_{n m}^{\prime}$ denote the total number of distinct orders $\leq m$ of the tree-components of the graph $G(n, p)$, and let

$$
\mathscr{E}_{n m}^{\prime}=\sum_{j \leq m} \varepsilon_{n j}^{\prime},
$$

see (1.7) for the precise definition of the independent binary variables $\varepsilon_{n j}^{\prime}(-\infty<$ $j<\infty)$. Introduce $n_{1}=\left[n^{1 / 6}\right]$.

For every $z \in\left(z_{1}, 1\right)$, where $z_{1}=\max \left(e^{-\alpha / 2}, 1 / 2\right)$ and $\alpha=c-1-\log c$, we have

$$
E\left(z^{\mathscr{D}_{n n_{1}}^{\prime}-D_{n}}\right)-E\left(z^{\mathscr{E}_{n}^{\prime}}\right) \rightarrow 0, \quad n \rightarrow \infty .
$$


Before proving this lemma, let us show how it implies (2.64) for every $z \in$ $\left(z_{1}^{1 / 2}, 1\right)$. Define

$$
W_{n}(z)=z^{\mathscr{D}_{n}^{\prime}-D_{n}}, \quad W_{n n_{1}}(z)=z^{\mathscr{D}_{n n_{1}}^{\prime}-D_{n}}
$$

and let $I_{n}$ be the indicator of the event $\left\{W_{n}(z) \neq W_{n n_{1}}(z)\right\}$. Obviously, $W_{n n_{1}}(z) \geq$ $W_{n}(z)$, and a.s. these random variables are equal, because $(c<1$ !) a.s. $G(n, p)$ does not have a component of order $>n_{1}$. Since $W_{n}^{2}(z)=W_{n}\left(z^{2}\right)$, and $z^{2} \in\left(z_{1}, 1\right)$, we have

$$
\begin{aligned}
0 & \leq E\left[W_{n n_{1}}(z)-W_{n}(z)\right] \leq E\left[I_{n} W_{n n_{1}}(z)\right] \\
& \leq P^{1 / 2}\left(I_{n}=1\right) E^{1 / 2}\left[W_{n n_{1}}^{2}(z)\right]=o\left(E^{1 / 2}\left[W_{n n_{1}}^{\prime}\left(z^{2}\right)\right]\right) \\
& =o\left(E^{1 / 2}\left[\left(z^{2}\right)^{\mathscr{E}_{n}^{\prime}}\right]\right)+o(1)=o(1), \quad n \rightarrow \infty .
\end{aligned}
$$

(We have used the fact that $\mathscr{E}_{n}^{\prime}$ is bounded in probability.)

ProOF OF LEMMA 7. Fix an integer $m>0$. On the event $A_{n}(j, \kappa)$, see (2.33),

$$
\mathscr{D}_{n m}^{\prime}=\sum_{r \leq m} g_{r}\left(j_{r}\right)
$$

here $g_{r}(l)=0$ for $r>m, l \geq 0$, and if $r \leq m$, then $g_{r}(l)=0$ for $l=0$ and $g_{r}(l)=1$ for $l>0$. So, using again (2.35), (2.37) and also the fact that $z \leq 1$, we obtain similarly to (2.40) and (2.41):

$$
E\left(z^{\mathscr{D} n_{m}^{\prime}}\right)=O\left(n^{-3 / 4}\right)+\omega_{n} S_{n m}(z)
$$

where

$$
\begin{aligned}
S_{n m}(z) & =\operatorname{coeff}_{x^{n}} \prod_{r \leq m}\left\{1+z\left[\exp \left(f_{r}(x)\right)-1\right]\right\} \cdot \exp \left[\sum_{r^{\prime}>m} f_{r^{\prime}}(x)+u(x)\right] \\
& =\operatorname{coeff}_{x^{n}} T_{m}(x, z),
\end{aligned}
$$

and

$$
f_{r}(x)=(q / p) x^{r} t_{*}(r) / r !, \quad r \geq 1,
$$

(cf. Wilf [22, Equation 7]). Hence, for $m=n_{1}$,

$$
E\left(z^{\mathscr{D}_{n_{1}}^{\prime}}\right)=O\left(n^{-3 / 4}\right)+\omega_{n} S_{n}(z), \quad S_{n}(z)=(2 \pi i)^{-1} \int_{C} x^{-(n+1)} T(x, z) d x
$$

here $T(x, z)=T_{n_{1}}(x, z)$ and $C$ is again a circle of radius $\rho<e^{-1}$. Set $x=\rho e^{i \theta}$, $\theta \in(-\pi, \pi]$ and, as in the proof of Lemma 6 , break the integral in two integrals, over $C_{1}=\left\{x=\rho e^{i \theta},|\theta| \leq n^{-5 / 12}\right\}$ and over $C_{2}=C \backslash C_{1}$.

(a) $|\theta|<n^{-5 / 12}$. In this case, for every $r \leq n_{1}\left(=\left[n^{1 / 6}\right]\right)$, and large enough $n$,

$$
\operatorname{Re}\left(x^{r}\right)=\rho^{r} \cos (r \theta) \geq \rho^{r} \cos \left(n_{1} \cdot n^{-5 / 12}\right) \geq \rho^{r} / 2,
$$

so

$$
\left|\exp \left(f_{r}(x)\right)\right|=\exp \left(\operatorname{Re} f_{r}(x)\right) \geq \exp \left[f_{r}(\rho) / 2\right] \geq 1 .
$$

Further, notice that

$$
1+z\left(e^{f_{r}}-1\right)=e^{f_{r}}\left[1-(1-z)\left(1-e^{-f_{r}}\right)\right] \quad\left(f_{r}=f_{r}(x)\right),
$$


B. PitTel

where, by (2.70) and $z>1 / 2$,

$$
\left|(1-z)\left(1-e^{-f_{r}}\right)\right| \leq 2(1-z)<1 .
$$

Subsequently, see (2.67)-(2.69),

$$
(2 \pi i)^{-1} \int_{C_{1}} x^{-(n+1)} T(x, z) d x=(2 \pi)^{-1} \int_{|\theta|<n^{-5 / 12}} \exp (K(x, z))(i x)^{-1} d x
$$

where

$$
\begin{gathered}
K(x, z)=(q / p) \tau_{*}(x)+u(x)-n \log x+L(x, z) \\
L(x, z)=\sum_{r \leq n_{1}} \log \left[1-(1-z)\left(1-e^{-f_{r}}\right)\right]
\end{gathered}
$$

and there is used the principal branch of the logarithmic function, see (2.71).

Choose $\rho$ a positive real root of $K_{x}(x, z)=0$, that is (see (2.73)),

$$
K_{x}(x, z)=c^{-1}(n-c) e^{\tau}-n / x+u_{x}+L_{x}(x, z)=0 .
$$

To prove that $(2.75)$ has a root $\rho$ which is close to $\bar{x}=c e^{-c}$, consider $x \in\left(\rho_{1}, \rho_{2}\right)$, where $0<\rho_{1}<\bar{x}<\rho_{2}<e^{-1}$. By (2.68), (2.71) and (2.74), rather crudely

$$
\begin{aligned}
\left|L_{x}(x, z)\right| & \leq(1-z)(2 z-1)^{-1} \sum_{r \leq n_{1}} e^{-f_{r}} f_{r}^{\prime} \quad\left(f_{r}^{\prime}=\frac{d f_{r}(x)}{d x}\right) \\
& \leq(1-z)(2 z-1)^{-1} \rho_{1}^{-1} \sum_{r \leq n_{1}} r e^{-f_{r}} f_{r} \\
& \leq(1-z)(2 z-1)^{-1} \rho_{1}^{-1} e^{-1} n_{1}^{2}=O\left(n^{1 / 3}\right) \quad\left(f e^{-f} \leq e^{-1}\right) .
\end{aligned}
$$

Also $u_{x}=O(1)$; so using (2.46) again, we obtain: there exists a root $\rho \in\left(\rho_{1}, \rho_{2}\right)$ such that

$$
\rho=\bar{x}+O\left(n^{-1} n^{1 / 3}\right)=\bar{x}+O\left(n^{-2 / 3}\right) .
$$

Furthermore, arguing as in (2.76) and applying (2.70), we can easily show that for $|\theta| \leq n^{-5 / 12}$,

$$
\begin{aligned}
\left|L_{x x}(x, z)\right| & =O\left(\sum_{r \leq n_{1}} r^{2} e^{-f_{r} / 2} f_{r}\right) \quad\left(f_{r}=f_{r}(\rho)\right) \\
& =O\left(n_{1}^{3}\right)=O\left(n^{1 / 2}\right),
\end{aligned}
$$

or, $u_{x x}=O(1)$

$$
K_{x x}(x, z)=n / \bar{x}^{2}(1-c)+O\left(n^{7 / 12}\right) .
$$

Since $K_{x}(\rho, z)=0$, we have then (cf. $\left.(2.54)-(2.56)\right)$ :

$$
\begin{aligned}
K(x, z) & =K(\rho, z)-[n /(1-c)] \theta^{2} / 2+O\left(n^{-1 / 4}\right) \\
K(\rho, z) & =K(\bar{x}, z)+\left[\left(c^{-1} e^{\bar{\tau}}-1 / \bar{x}\right) n+O\left(n^{1 / 3}\right)\right](\rho-\bar{x})+O\left(n^{-1 / 4}\right) \\
& =K(\bar{x}, z)+O\left(n^{-1 / 4}\right) .
\end{aligned}
$$

Thus, see (2.67),

$$
(2 \pi i)^{-1} \int_{C_{1}} x^{-(n+1)} T(x, z) d x=[2 \pi n /(1-c)]^{-1 / 2} \exp (K(\bar{x}, z))(1+o(1))
$$


(b) $\theta \geq n^{-5 / 12}$. To estimate the integral over $C_{2}$, we need the following interesting inequality (cf. [15, Equation A1]): for $z \geq 1 / 2$,

$$
\left|1+z\left(e^{y}-1\right)\right| \leq \exp [(\operatorname{Re} y-|y|) / 2]\left[1+z\left(e^{|y|}-1\right)\right] .
$$

(We will prove it later.) Now, for $\theta$ 's in question

$$
\operatorname{Re} f_{1}(x)-\left|f_{1}(x)\right|=(q / p) \rho(\cos \theta-1) \leq-\chi n \theta^{2} \leq-\chi n^{1 / 6} .
$$

According to (2.67) and (2.68), we have then

$$
\begin{aligned}
\left|x^{-n} T(x, z)\right| & \leq \exp \left(-\chi n^{1 / 6} / 2\right) \rho^{-n} T(\rho, z)=\exp \left[K(\rho, z)-\chi n^{1 / 6} / 2\right] \\
& \leq \exp \left[K(\bar{x}, z)-\chi n^{1 / 6} / 3\right],
\end{aligned}
$$

see (2.77). Thus, by (2.78),

$$
\left|(2 \pi i)^{-1} \int_{C_{2}} x^{-(n+1)} T(x, z) d x\right|=O\left(\exp \left[K(\bar{x}, z)-\chi n^{1 / 6} / 3\right]\right)=o\left(\left|\int_{C_{1}}\right|\right),
$$

and we conclude that

$$
(2 \pi i)^{-1} \int_{C} x^{-(n+1)} T(x, z) d x=\left[\frac{2 \pi n}{(1-c)}\right]^{-1 / 2} \exp (K(\bar{x}, z))(1+o(1)) .
$$

(c) It remains to evaluate $K(\bar{x}, z)$. Since $\tau\left(c e^{-c}\right)=c$, by (2.1), (2.38) and (2.73), we get

$$
\begin{aligned}
K(\bar{x}, z)= & c^{-1}(n-c)\left(c-c^{2} / 2\right)+\log (1-c)^{-1 / 2} \\
& -\left(c / 2+c^{2} / 4\right)-n \log \left(c e^{-c}\right)+L(\bar{x}, z),
\end{aligned}
$$

so turn now to $L(\bar{x}, z)$. Recalling that $D_{n}=\left[s_{n}\right]$, where

$$
s_{n}=\alpha^{-1} \log \left[\alpha^{5 / 2} n / c(2 \pi)^{1 / 2} \log ^{5 / 2} n\right], \quad \alpha=c-1-\log c
$$

(thence $D_{n}=o\left(n_{1}\right)$ ), we can write

$$
\begin{aligned}
L(\bar{x}, z) & =\sum_{r \leq n_{1}} \log \left[1-(1-z)\left(1-e^{-f_{r}}\right)\right] \\
& =\sum_{r \leq D_{n}}+\sum_{D_{n}<r \leq n_{1}}=\sum_{1}+\sum_{2} .
\end{aligned}
$$

Here

$$
\sum_{1}=D_{n} \log z+\sum_{r \leq D_{n}} \log \left[z^{-1} e^{-f_{r}}+\left(1-e^{-f_{r}}\right)\right]
$$

and, for every fixed $j=D_{n}-r$,

$$
\begin{aligned}
f_{r} & =c^{-1}(n-c) \bar{x}^{r} t_{*}(r) / r ! \sim c^{-1} n(2 \pi)^{-1 / 2} r^{-5 / 2}(e \bar{x})^{r} \\
& \sim c^{-1}(2 \pi)^{-1 / 2} n D_{n}^{-5 / 2} \exp \left[-\alpha\left(D_{n}-j\right)\right] \\
& \sim\left[c^{-1}(2 \pi)^{-1 / 2} n s_{n}^{-5 / 2} \exp \left(-\alpha s_{n}\right)\right] \exp \left[\alpha\left(d_{n}+j\right)\right] \quad\left(d_{n}=s_{n}-\left[s_{n}\right]\right) \\
& \sim \exp \left[\alpha\left(d_{n}+j\right)\right] .
\end{aligned}
$$

So,

$$
\begin{gathered}
\sum_{1}=D_{n} \log z+\sum_{j \geq 0} \log \left[z^{-1} p_{n j}+\left(1-p_{n j}\right)\right]+o(1), \quad n \rightarrow \infty \\
p_{n j}=\exp \left\{-\exp \left[\alpha\left(d_{n}+j\right)\right]\right\}
\end{gathered}
$$


Similarly,

$$
\sum_{2}=\sum_{j<0} \log \left[p_{n j}+z\left(1-p_{n j}\right)\right]+o(1), \quad n \rightarrow \infty .
$$

Collecting together (2.80)-(2.83), we obtain

$$
\begin{aligned}
(2 \pi i)^{-1} & \int_{C} x^{-(n+1)} T(x, z) d x \\
\sim & (2 \pi n)^{-1 / 2}(e / c)^{n} \exp \left[n c / 2-3 c / 2+c^{2} / 4\right] \\
& \cdot z^{D_{n}} \prod_{j<0} \log \left[p_{n j}+z\left(1-p_{n j}\right)\right] \prod_{j \geq 0} \log \left[z^{-1} p_{n j}+\left(1-p_{n j}\right)\right] .
\end{aligned}
$$

Thus finally, by (2.60) and (2.69),

$$
\begin{aligned}
E\left(z^{\mathscr{D}_{n_{1}}}\right)= & z^{D_{n}} \prod_{j<0} \log \left[p_{n j}+z\left(1-p_{n j}\right)\right] \\
& \prod_{j \geq 0} \log \left[z^{-1} p_{n j}+\left(1-p_{n j}\right)\right](1+o(1))+O\left(n^{-3 / 4}\right) .
\end{aligned}
$$

This leads to (2.64), since $D_{n} \sim \alpha^{-1} \log n$ and $z \geq e^{-\alpha / 2}$.

Let us prove the inequality (2.79). We have

$$
1+z\left(e^{y}-1\right)=z e^{y / 2}\left[e^{y / 2}+z^{-1}(1-z) e^{-y / 2}\right]=z e^{y / 2} \sum_{j \geq 0} c_{j} y^{j}
$$

where

$$
c_{j}=\left[1+z^{-1}(1-z)(-1)^{j}\right] / 2^{j} j ! \geq \frac{\left[1-z^{-1}(1-z)\right] / 2^{j}}{j !}>0,
$$

since $z>1 / 2$. Therefore,

$$
\begin{aligned}
\left|1+z\left(e^{y}-1\right)\right| & \leq z \exp (\operatorname{Re} y / 2) \sum_{j \geq 0} c_{j}|y|^{j} \\
& =\exp \left[\frac{\operatorname{Re} y-|y|}{2}\right] z \exp \left(\frac{|y|}{2}\right) \sum_{j \geq 0} c_{j}|y|^{j} \\
& =\exp [(\operatorname{Re} y-|y|) / 2]\left[1+z\left(e^{|y|}-1\right)\right] . \quad \square
\end{aligned}
$$

Theorem 4 is proved.

Note. Again, our proof relies heavily on the condition $c<1$, but it seems plausible that Theorem 4 holds true for $c>1$ as well.

ACKNOWLEDGMENT. I gratefully acknowledge my debt to Béla Bollobás for allowing me to read and to use for teaching a typewritten version of his book on random graphs.

\section{REFERENCES}

1. M. Ajtai, J. Komlós, and E. Szemerédi, The longest path in a random graph, Combinatorica 1 (1981), 1-12.

2. A. D. Barbour, Poisson convergence and random graphs, Math. Proc. Cambridge Philos. Soc. 92 (1982), 349-359.

3. C. Berge, Principles of combinatorics, Academic Press, New York, 1971. 
4. B. Bollobas, Long paths in sparse random graphs, Combinatorica 2 (1982), 223-228.

5. B. Bollobás, T. I. Fenner and A. M. Frieze, Long cycles in sparse random graphs, Graph Theory and Combinatorics, Proc. Cambridge Combinatorial Conf. in honour of Paul Erdös, Academic Press, New York, 1984, pp. 59-64.

6. B. Bollobás, Random graphs, Academic Press, New York, 1985.

7. I. M. Curtiss, A note on the theory of moment generating functions, Ann. Math. Statist. 13 (1942), 430-433.

8. P. Erdós and A. Rényi, On random graphs. I, Publ. Math. Debrecen 6(1959), 290-297.

9.

10. F. Harary and E. M. Palmer, Graphical enumeration, Academic Press, New York, 1973.

11. L. Katz, Probability of indecomposability of a random mapping function, Ann. Math. Statist. 26 (1955), 512-517.

12. J. W. Moon, Counting labelled trees, Canadian Math. Congress, Clowes, London, 1970.

13. A. M. Odlyzko and L. B. Richmond, On the number of distinct block sizes in partitions of a set, J. Combin. Theory Ser. A 38 (1985), 179-181.

14. B. Pittel, On the probable behavior of some algorithms for finding the stability number of a graph, Math. Proc. Cambridge Philos. Soc. 92 (1982), 511-526.

15. $\ldots$, Paths in a random digital tree: limiting distributions, Adv. in Appl. Probab. 18 (1986), 139-155.

16. A. Rényi, Some remarks on the theory of trees, Publ. Math. Inst. Hungar. Acad. Sci. 4 (1959), 73-85.

17. A. Renyi and G. Szekeres, On the height of trees, J. Austral. Math. Soc. 7 (1967), 497-507.

18. J. Riordan, The enumeration of trees by height and diameter, IBM J. Res. Develop. 4 (1960), 473-478.

19. V. N. Sachkov, Probabilistic methods in combinatorial analysis, "Nauka", Moscow, 1978. (Russian)

20. G. Szekeres, Regular iteration of real and complex functions, Acta Math. 100 (1958), 103-258.

21. W. F. de la Vega, Long paths in random graphs, Studia Sci. Math. Hungar. 14 (1979), 335-340.

22. H. S. Wilf, Three problems in combinatorial asymptotics, J. Combin. Theory Ser. A 35 (1983), 199-207.

23. E. M. Wright, The number of connected sparsely edged graphs, J. Graph Theory 1 (1977), 317-330. 43210

Department of Mathematics, The Ohio State University, Columbus, Ohio

Current address: Computer Science Department, Stanford University, Stanford, California 94305 